Draft Version April 2, 2018

Preprint typeset using $\mathrm{IAT}_{\mathrm{E}} \mathrm{X}$ style emulateapj v. 5/2/11

\title{
HST-COS OBSERVATIONS OF AGN. I. ULTRAVIOLET COMPOSITE SPECTRA OF THE IONIZING CONTINUUM AND EMISSION LINES
}

\author{
J. Michael Shull, Matthew Stevans, \& Charles W. Danforth \\ CASA, Department of Astrophysical \& Planetary Sciences, \\ University of Colorado, Boulder, CO 80309 \\ Draft version April 2, 2018
}

\begin{abstract}
The ionizing fluxes from quasars and other active galactic nuclei (AGN) are critical for interpreting the emission-line spectra of AGN and for photoionization and heating of the intergalactic medium. Using ultraviolet spectra from the Cosmic Origins Spectrograph (COS) on the Hubble Space Telescope $(H S T)$, we have directly measured the rest-frame ionizing continua and emission lines for 22 AGN. Over the redshift range $0.026<z<1.44$, COS samples the Lyman continuum and many far-UV emission lines (Ly $\alpha \lambda 1216$, C IV $\lambda 1549$, Si IV/O IV] $\lambda 1400$, N V $\lambda 1240$, O VI $\lambda 1035$ ). Strong EUV emission lines with 14-22 eV excitation energies (Ne VIII $\lambda \lambda 770,780$, Ne V $\lambda 569$, O II $\lambda 834$, O III $\lambda 833, \lambda 702$, O IV $\lambda 788,608,554$, O V $\lambda 630, \mathrm{~N}$ III $\lambda 685$ ) suggest the presence of hot gas in the broad emission-line region. The rest-frame continuum, $F_{\nu} \propto \nu^{\alpha_{\nu}}$, shows a break at wavelengths $\lambda<1000 \AA$, with spectral index $\alpha_{\nu}=-0.68 \pm 0.14$ in the FUV (1200-2000 $\AA$ ) steepening to $\alpha_{\nu}=-1.41 \pm 0.21$ in the EUV (500-1000 $\AA$ ). The COS EUV index is similar to that of radio-quiet AGN in the 2002 $H S T$ /FOS survey $\left(\alpha_{\nu}=-1.57 \pm 0.17\right)$. We see no Lyman edge $\left(\tau_{\mathrm{HI}}<0.03\right)$ or He I $\lambda 584$ emission in the AGN composite. Our 22 AGN exhibit a substantial range of FUV/EUV spectral indices and a correlation with AGN luminosity and redshift, likely due to observing below the $1000 \AA$ break.

Subject headings: galaxies: active — line: profiles — quasars: emission lines - ultraviolet: galaxies
\end{abstract}

\section{INTRODUCTION}

Active galactic nuclei (AGN) hold a place of special importance in astrophysics, as examples of processes of high-energy emission and gas accretion around supermassive black holes and for their impact on surrounding gas in the nuclei of galaxies and throughout the intergalactic medium (IGM). Their rest-frame Lyman continuum (LyC) emission $(\lambda \leq 912 \AA)$ is likely to be the dominant source of metagalactic ionizing radiation over most of the age of the universe. The shape of their ionizing continua is critical for interpreting their broad emissionline spectra, and provides the ionization corrections required to derive abundances of the common (C IV, Si IV, $\mathrm{O}$ VI) metal-line systems in quasar absorption spectra (Schaye et al. 2003; Aguirre et al. 2004; Danforth \& Shull 2008). The AGN ionizing continua at 1 ryd and 4 ryd are also important for interpreting fluctuations in the ratio of the Ly $\alpha$ absorbers of H I and He II (Fardal et al. 1998; Fechner et al. 2006; Shull et al. 2004, 2010).

The nuclei of many galaxies exhibit emission lines characteristic of photoionization, both from hot stars and non-thermal continua. In a subset, the emission lines exhibit a range of ionization states and line widths uncharacteristic of normal or starburst galaxies. These AGN include objects classified as Seyfert galaxies, quasars (quasi-stellar radio galaxies), and QSOs (quasi-stellar objects). Their emission lines are believed to be produced by a photoionizing continuum extending from the extreme-ultraviolet to the X-ray, which may be produced by an accretion disk around the black hole and by relativistic electrons, involving synchrotron emission and Compton scattering. The ionizing spectrum extends to energies well beyond the hydrogen edge ( $h \nu \geq 1 \mathrm{ryd})$, in order to produce strong emission lines (Krolik \& Kallman 1988) from high ions such as Si IV (2.46 ryd), C IV (3.51 ryd), He II (4.00 ryd), N V (5.69 ryd), and O VI (8.37 ryd).

Although AGN are observed over a wide variety of luminosities and physical circumstances (mass, viewing angle, accretion rate), they display a consistent mean spectral energy distribution (SED) in the UV-optical. Individual sources exhibit considerable variations in the far ultraviolet (FUV, 1000-2000 А) and extreme ultraviolet (EUV, $\lambda<912 \AA$ ), with observed flux distributions fitted to power laws, $F_{\lambda} \propto \lambda^{\alpha_{\lambda}}$ and $F_{\nu} \propto \nu^{\alpha_{\nu}}$, where $\alpha_{\nu}=-\left(2+\alpha_{\lambda}\right)$. The FUV and EUV spectra afford a glimpse of the inner workings of the black hole accretion disk and the broad emission-line region (BELR). Ground-based surveys of AGN at $z>2$ have characterized the rest-frame ultraviolet, longward of the AGN Ly $\alpha$ emission line and free of intergalactic hydrogen absorption. Composite UV spectra of AGN have been compiled by many groups (Francis et al. 1991; Schneider et al. 1991; Carballo et al. 1999; Brotherton et al. 2001; Vanden Berk et al. 2001). All find a wide range of spectral indices, steepening toward high frequencies, with possible dependence on sample redshift and luminosity range.

The best direct probe of the rest-frame FUV and EUV continua comes from ultraviolet (space-borne) observations of AGN at $z \leq 1.5$. Although the rest-frame continuum shortward of $1216 \AA$ is accessible from the ground toward higher-redshift AGN, it is often complicated by intergalactic $\operatorname{Ly} \alpha$ absorption (Fan et al. 2001). Thus, ultraviolet spectrographs aboard the International Ultraviolet Explorer (IUE), Far Ultraviolet Spectroscopic Exnloron FISE) and Hubble Space Telescope $(H S T)$ have
anforth@colorado.edu

michael.shull@colorado.edu, matthew.stevans@colorado.edu, charles.dannorth@coloradò.edu 
provided access to the AGN continuum below Ly $\alpha$ in a number of bright, low- $z$ targets. Composite spectra of these AGN were created from HST/FOS and HST/GHRS observations (Zheng et al. 1997; Telfer et al. 2002) and from FUSE (Scott et al. 2004). For 184 QSOs at $z>0.33$ studied with $H S T / F O S$, Telfer et al. (2002) fitted the composite continuum (500-1200 $)$ as a power law with spectral index $\alpha_{\nu}=-1.76 \pm 0.12$. For a sub-sample of 39 radio-quiet QSOs, they found $\alpha_{\nu}=-1.57 \pm 0.17$. In contrast, the FUSE survey of 85 AGN at $z \leq 0.67$ (Scott et al. 2004) found a harder composite spectrum with $\alpha_{\nu}=-0.56_{-0.28}^{+0.38}$ and suggested that the difference may reflect a luminosity dependence through the well-known anti-correlation (Baldwin effect) between emission-line strength and AGN luminosity. The difference could also arise from the small numbers of targets observed by the different instruments; fewer than 10 AGN observations cover the spectral range $450 \AA \lesssim \lambda \lesssim 600 \AA$. In all of these studies, a crucial issue is the placement of the EUV continuum, which sits below strong emission lines such as Ne VIII, Ne V, O III, $\mathrm{O}$ IV, O V, and O VI.

Figure 1 shows current estimates of the metagalactic ionizing continuum, interpolated between far-UV and Xray energies. At the Lyman limit (1 ryd), we adopt a specific intensity $I_{\nu} \approx 2 \times 10^{-23} \mathrm{erg} \mathrm{cm}^{-2} \mathrm{~s}^{-1} \mathrm{~Hz}^{-1} \mathrm{sr}^{-1}$ (Shull et al. 1999; Haardt \& Madau 2012) and use the two "bow-tie" extrapolations derived in the composite AGN spectra from $H S T$ and FUSE. In the X-ray band $(0.5-2 \mathrm{keV})$, we use background estimates from ROSAT measurements of AGN in the Lockman Hole (Hasinger et al. 1993) normalized at $1 \mathrm{keV}$ with energy flux $F_{E} \propto E^{-0.96 \pm 0.11}$. The ionizing intensity in the mid-EUV remains uncertain, depending on how one interpolates between the measured fluxes at 1.0-1.5 ryd and $0.5-2 \mathrm{keV}$.

In this paper, we chose an initial COS sample of 22 AGN (non-blazar) at $z \leq 1.5$, available in 2011 January and selected to have $\overline{\mathrm{S}} / \mathrm{N}$ sufficient to directly measure their rest-frame UV continua and emission lines. With minimal contamination by Ly $\alpha$-forest absorption, we develop a composite AGN spectrum in the FUV and EUV for targets at redshifts between $z=0.026$ and $z=1.44$. We identify the prominent AGN emission lines and line-free portions of the spectrum and fit the underlying continua below the emission lines, excluding interstellar and intergalactic absorption lines. In Section 2 we describe the COS data reduction, our techniques for flux-alignment and patching $H S T$ /COS spectra with earlier UV spectra taken by IUE and FUSE. In Section 3 we describe our results on the individual and composite spectra. Section 4 presents our conclusions and their implications.

\section{OBSERVATIONS OF ULTRAVIOLET SPECTA OF AGN}

\subsection{Data Acquisition and Reduction Methods}

Table 1 lists the relevant COS observational parameters of 22 AGN (non-blazar) targets, selected in 2011 January as the best available high-S/N spectra of AGN. This initial sample was not intended to be archivally complete, as we discuss at the end of Section 2. Most of the low-redshift AGN are Seyfert galaxies. Several AGN exhibit narrow intrinsic absorption lines (e.g., Mrk817,

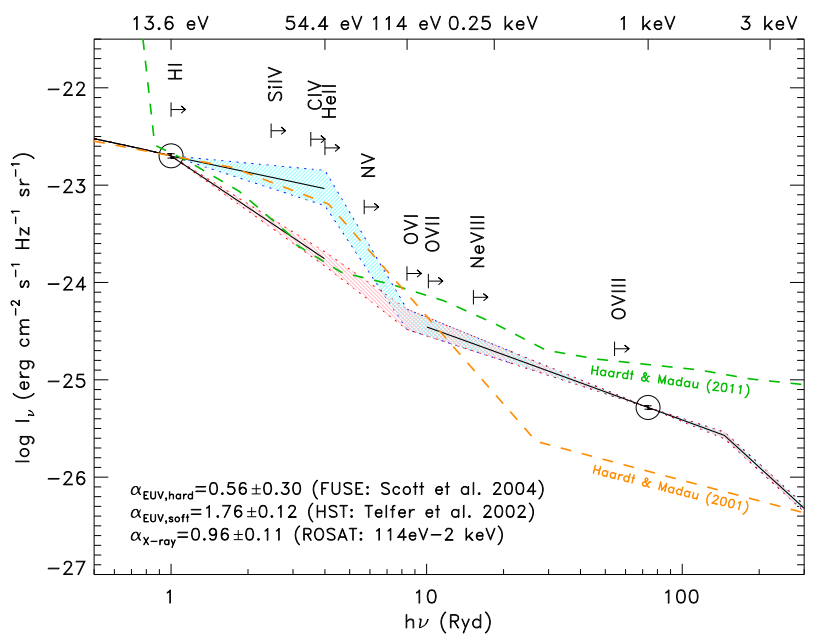

FIG. 1. - Two possible model spectra of AGN ionizing radiation (EUV to X-ray), normalized at 1 ryd with specific intensity $I_{0}=2 \times 10^{-23} \mathrm{erg} \mathrm{cm}^{-2} \mathrm{~s}^{-1} \mathrm{~Hz}^{-1} \mathrm{sr}^{-1}$ and two different flux distributions, $F_{\nu} \propto \nu^{\alpha_{\nu}}$, between 1 and 4 ryd. Previous composite AGN spectra are shown in red (HST) and light blue (FUSE), with slopes $\alpha_{\nu}=-1.76 \pm 0.12$ (Telfer et al. 2002) and $\alpha_{\nu}=-0.56_{-0.28}^{+0.38}$ (Scott et al. 2004). Soft X-ray data $(0.5-2 \mathrm{keV})$ are from ROSAT observations of AGN in the Lockman Hole (Hasinger et al. 1993; Hasinger 1994) normalized at $E=1 \mathrm{keV}$ (73.53 ryd) with specific energy flux $F_{E} \propto E^{-0.96 \pm 0.11}$. Ionization potentials of $\mathrm{H}$ I and He II and energies required to produce key metal ions are shown as arrows. Metagalactic backgrounds from Haardt \& Madau (2001, 2012) are also shown.

Mrk 290, Mrk 1513, PG 1115+407). With the spectral resolution and high throughput of COS, we are able to fit out all these absorbers and measure the underlying continuum. All targets were observed in both the G130M

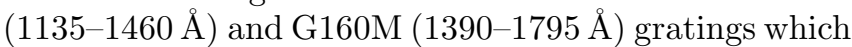
provide medium resolution $(R \equiv \lambda / \Delta \lambda \approx 18,000$ or $\left.\Delta v \approx 17 \mathrm{~km} \mathrm{~s}^{-1}\right)$. The COS instrument and data acquisition are described by Osterman et al. (2011) and Green et al. (2012). The NUV-imaging target acquisitions were performed with the MIRRORA/PSA mode. The COS primary science aperture $\left(\sim 2.5^{\prime \prime}\right.$ diameter $)$ yields good centering to maximize the COS throughput and spectral resolving power.

Each sight line was observed with four or more exposures in both the G130M and G160M gratings. The central wavelengths were shifted between exposures to dither instrumental features in wavelength space and to provide a continuous spectrum over the entire FUV spectral range. Individual exposures for each sight line were retrieved from the Multi-mission Archive for Space Telescope (MAST) processed with the default CALCOS reduction pipeline. Alignment and coaddition of the individual exposures was carried out using IDL routines developed by the COS GTO team specifically for FUV spectra (IDL routines are available at http://casa.colorado.edu/\$ \sim\$danforth/costools.html.) Danforth et al. (2010) describe the process in detail; the only significant difference is our omission of the pseudo flatfielding procedure from that paper. A similar procedure is now included in the standard CALCOS reduction. Data coalignment was achieved with strong interstellar features in each exposure, cross-correlated and interpolated onto a common wavelength scale. The wavelength shifts were typically on the order of a resolu- 
tion element $(\sim 0.1 \AA)$ or less. The coadded flux at each wavelength was taken to be the exposure-weighted mean of flux in each aligned exposure. Exposure times at the edges of detector segments were adjusted downward to underweight these regions.

\subsection{Fitting and Patching the UV Spectra}

Here, we discuss how we aligned the fluxes, dealt with detector gaps, dereddening, Lyman-limit systems, cosmological factors in rest-frame wavelength and specific flux, and other technical issues. We then provide details of how we constructed the composite spectra, with brief discussion of the order of adding spectra and choosing wavelength-pivot points. To combine the nonsimultaneous observations from FUSE, HST/COS, and $I U E$, we scale the flux within the regions of spectral overlap to agreement. The FUSE and COS spectra overlap by $\sim 45 \AA$, those of COS and IUE by $\sim 645 \AA$, and those of IUE-SWP (short-wavelength-prime channel) and IUELWR (long-wavelength-redundant channel) by $\sim 125 \AA$. When the overlapping regions contain uncontaminated continuum flux, we use only these regions to scale the data. The FUSE and IUE data are scaled to $H S T / C O S$ because, to our knowledge, the flux calibration of the $H S T /$ COS spectra is more reliable. The IUE-SWP and IUE-LWR fluxes appear consistent and are not scaled to each other. Scaling factors for the IUE-to-COS spectra range from 0.64 to 2.07 with an average of 1.13 , and for the FUSE-to-COS data from 0.35 to 1.38 with an average of 0.90 .

Source variability could affect the absolute flux and shape of AGN SEDs in the ultraviolet. For example, IUE-AGN variability campaigns found that the UV continua hardened as the flux increased for the Seyfert galaxies NGC 4151 (Crenshaw et al. 1996; Edelson et al. 1996), NGC 5548 (Clavel et al. 1991), and NGC 3783 (Reichert et al. 1994). For Fairall 9 (Rodriguez-Pascual et al. 1997) and NGC 3516 (Edelson et al. 2000) there was no significant change in the relative FUV flux. Determining the actual shape of the underlying UV continuum was challenging in these studies, owing to spectral dilution by the Fe II pseudo-continuum (NUV) and difficulty finding line-free continuum windows. The combination of recent and archival data could make our SEDs vulnerable to this effect. However, because we primarily use coeval COS data to measure the slope of the continuum, our scaling should not greatly influence the result.

As noted earlier, several of our Seyferts and AGN show outflows and intrinsic absorption common in Seyfert galaxies and QSOs (Crenshaw et al. 2003). In the seven low- $z$ targets $(z \leq 0.154)$ the C IV line provides the best indicator of such outflows (Mrk 817, Mrk 290, NGC 1513, PG 1115+407). At higher redshift, C IV shifts out of the COS bands, but one can use O VI $\lambda 1035$ as an indicator (e.g., HE 0153-4520, HE 0226-4110, HE 1102+3441). For the highest-redshift AGN, one can use Ne VIII $\lambda \lambda 770$, 780 to probe outflows. For our continuum fitting, we are able to fit out all the absorbers: intrinsic systems as well as interstellar and intergalactic lines. This capability is made possible by the high-S/N and highresolution of COS. We also mask out Galactic Ly $\alpha$ absorption (1215.67 $\AA$ ) and the geocoronal emission line of O I $\lambda 1304$ in every spectrum.
We correct the spectra for Galactic reddening, using the empirical mean extinction curve of Fitzpatrick (1999) with a ratio of total-to-selective extinction $R_{v}=$ $A_{V} / E(B-V)=3.1$ and color excesses $E(B-V)$ from NEDT based on dust mapping by Schlegel et al. (1998). For our purposes, this extinction curve is extrapolated down to $1000 \AA$. If we use the extinction curve of Cardelli et al. (1989), the spectral index changes by only $\Delta \alpha \approx 0.02$. We inspect the spectra for absorption from Lyman limit systems (LLS) and partial LLS by identifying many Lyman lines that are blueshifted from Galactic absorption. Table 2 shows the strengths (column densities $N_{\mathrm{HI}}$ ) and redshifts of 17 LLS found toward 8 AGN in our sample. We account for Lyman continuum absorption by correcting for the $\nu^{-3}$ opacity shortward of each Lyman edge using the optical depth, $\ln \left(F_{+} / F_{-}\right)$, where $F_{-}$is the median flux of the pixels just blueward of the break and $F_{+}$is the median flux of the pixels in selected windows longward of the break. We have used the restored continua in all cases except for the strong LLS toward SBS $1108+560\left(\log N_{\mathrm{HI}}=17.78\right)$, for which we ignore the spectrum below $755 \AA$ (rest frame). Finally, we apply redshift corrections to shift each spectrum to the AGN rest frame by dividing the wavelengths by $(1+z)$.

We use the IDL routine MPFIT 2 to fit the continuum of each spectrum with a power law of form, $F_{\lambda} \propto \lambda^{\alpha_{\lambda}}$, with spectral indices, $\alpha_{\lambda}$, listed in Table 1. Because of the strong emission lines commonly seen in AGN spectra, finding windows of completely uncontaminated continuum can be difficult. We focus on wavelength regions with the lowest relative flux (local minima) that appear to be free of emission lines. We avoid the prominent emission from Ly $\alpha 1216$ and the doublets of C IV $\lambda \lambda 1548,1551, \mathrm{~N}$ V $\lambda \lambda 1239,1243$, O VI $\lambda \lambda 1032,1038$, and Si IV $\lambda \lambda 1394,1403$. The Si IV lines are typically blended with O IV] $\lambda 1405$, and the O VI lines with $\operatorname{Ly} \beta \lambda 1025$. We also detect a number of weaker lines, known from atomic data and clearly seen in Figure 2 in high-S/N spectra of Mrk 817 and Mrk 1513. These include line blends of O I $\lambda 1304$ and Si II $\lambda 1308$, He II $\lambda 1640$, O III] $\lambda 1605$, C II $\lambda 1335$, N II $\lambda 1085$, Fe III $\lambda 1126$, and N IV] $\lambda 1486$. These features appear at the expected wavelengths, although in some cases residual flux from these blends appears as a pseudo-continuum. The high resolution of $\left(\sim 20 \mathrm{~km} \mathrm{~s}^{-1}\right)$ and sensitivity of COS (S/N listed in Table 1) allow us to exclude many absorption lines from the Galactic interstellar medium and IGM. The most common regions of (rest-frame, line-free) continuum (listed here in $\AA$ ) include: $660-670,715-735,860$ 880, 1090-1105, 1140-1155, 1280-1290, 1315-1325, and $1440-1465$.

1 NASA/IPAC Extragalactic Database (NED) is operated by the Jet Propulsion Laboratory, California Institute of Technology, under contract with the National Aeronautics and Space Administration, http://nedwww.ipac.caltech.edu.

2 http://nedwww.ipac.caltech.edu (see also Markwardt 2009) 
TABLE 1

COS Observations (22 AGN TARgets)

\begin{tabular}{|c|c|c|c|c|c|c|c|}
\hline $\begin{array}{l}\text { AGNa }^{a} \\
\text { Target }\end{array}$ & $\begin{array}{l}\text { AGNa }^{a} \\
\text { Type }\end{array}$ & $\overline{z^{\mathrm{a}}}$ & $\alpha_{\lambda}$ & $\begin{array}{c}F_{0}^{\mathrm{a}} \\
\left(1100^{\AA}\right)\end{array}$ & $\begin{array}{c}F_{\lambda}{ }^{\mathrm{a}} \\
(1300 \AA)\end{array}$ & $\begin{array}{l}\log \left(\lambda L_{\lambda}\right)^{\mathrm{a}} \\
(1100 \AA)\end{array}$ & $(\mathrm{S} / \mathrm{N})_{\mathrm{res}}{ }^{\mathrm{a}}$ \\
\hline Mrk 335 & Sy 1 & 0.025785 & -1.35 & 5.68 & 4.0 & 43.98 & 71,18 \\
\hline Mrk 290 & Sy 1.5 & 0.029577 & -1.05 & 1.80 & 1.9 & 43.60 & 63,26 \\
\hline Mrk 817 & Sy 1.5 & 0.031455 & -1.49 & 9.51 & 10.0 & 44.38 & 73,36 \\
\hline PG 1011-040 & Sy 1.2 & 0.058314 & -1.75 & 2.75 & 3.2 & 44.39 & 38,21 \\
\hline Mrk 1513 & Sy 1.5 & 0.062977 & -1.09 & 2.73 & 5.7 & 44.46 & 36,8 \\
\hline Mrk 876 & Sy 1 & 0.129 & -1.19 & 4.90 & 3.8 & 45.38 & 72,55 \\
\hline PG $1115+407$ & Sy 1 & 0.1546 & -1.42 & 1.07 & 1.0 & 44.89 & 27,20 \\
\hline HE $0153-4520$ & QSO & 0.451 & -0.80 & 1.36 & 1.4 & 46.06 & 33,29 \\
\hline PG $1259+593$ & Sy 1 & 0.4778 & -1.09 & 1.18 & 1.4 & 46.06 & 43,35 \\
\hline HE $0226-4110$ & Sy 1 & 0.493368 & -1.47 & 1.73 & 2.0 & 46.26 & 49,38 \\
\hline HS $1102+3441$ & QSO & 0.5088 & -1.26 & 0.255 & 0.30 & 45.46 & 25,27 \\
\hline HE 0238-1904 & QSO & 0.631 & -0.42 & 1.48 & 1.3 & 46.45 & 38,26 \\
\hline $3 \mathrm{C} 263$ & Sy 1.2 & 0.6460 & -1.03 & 0.693 & 1.0 & 46.14 & 47,38 \\
\hline SBS1108+560 & QSO & 0.7666 & -0.84 & 0.422 & $0.013^{*}$ & 46.11 & 4,16 \\
\hline SBS $1122+594$ & QSO & 0.852 & -0.62 & 0.231 & 0.24 & 45.96 & 18,15 \\
\hline FBQS J0751+2919 & QSO & 0.9149 & -0.51 & 0.731 & 0.65 & 46.54 & 40,33 \\
\hline PG1407+265 & QSO & 0.95 & -1.56 & 0.671 & 1.1 & 46.55 & 66,36 \\
\hline PG1148+549 & QSO & 0.9754 & -0.53 & 0.375 & 0.47 & 46.32 & 41,33 \\
\hline HE 0439-5254 & QSO & 1.053 & -1.31 & 0.201 & 0.39 & 46.13 & 23,17 \\
\hline PG $1206+459$ & QSO & 1.1625 & -0.28 & 0.453 & 0.36 & 46.59 & 30,33 \\
\hline $\mathrm{PG} 1338+416$ & QSO & 1.214 & -1.17 & 0.0874 & 0.17 & 45.93 & 26,23 \\
\hline Q0232-042 & QSO & 1.437368 & -0.67 & 0.196 & 0.22 & 46.46 & 24,17 \\
\hline
\end{tabular}

a AGN targets, types, redshifts, fluxes, spectral indices, luminosities, and $\mathrm{S} / \mathrm{N}$ ratios. All fluxes in units of $10^{-14}$ erg cm ${ }^{-2} \mathrm{~s}^{-1} \AA^{-1}$. Rest-frame, dereddened spectral distributions are fitted to power laws, $F_{\lambda}=F_{0}(\lambda / 1100 \AA)^{\alpha}$. Wavelength index $\alpha_{\lambda}$ corresponds to frequency index $\alpha_{\nu}=-\left[2+\alpha_{\lambda}\right]$. The 22 AGN are listed in three redshift groups: low $\left(0.026 \leq z \leq 0.154\right.$ with $\left.\left\langle\alpha_{\lambda}\right\rangle=-1.33\right)$; intermediate $\left(0.45 \leq z \leq 0.77\right.$ with $\left.\left\langle\alpha_{\lambda}\right\rangle=-0.99\right)$; high $\left(0.85 \leq z \leq 1.44\right.$ with $\left.\left\langle\alpha_{\lambda}\right\rangle=-0.83\right)$. The eight columns show: (1) AGN target; (2) AGN type; (3) AGN redshift; (4) Fitted spectral index $\alpha_{\lambda}$; (5) Rest-frame flux normalization $F_{0}$ at $1100 \AA$; (6) Observed flux $F_{\lambda}$ at $1300 \AA$; (7) Band luminosity, $\lambda L_{\lambda}$ at $1100 \AA$ (in erg s ${ }^{-1}$ ); (8) signal-to-noise at $1250 \AA$ and $1550 \AA$ for data with G130M (1132-1460 $\AA$ ) and G160M (1394-1798 $\AA$ ) gratings, respectively. Flux at $1300 \AA$ for SBS $1108+560$ (noted with *) is low, owing to LyC absorption $(\lambda<1334 \AA$ ) from a Lyman limit system at $z=0.46335$.

\subsection{Constructing and Fitting the Composite Spectrum}

The spectra are resampled to wavelength bins of $0.1 \AA$, using the same procedure as in Telfer et al. (2002). Before resampling, we discard any pixels with $\mathrm{S} / \mathrm{N}<1$. The flux corresponding to each new wavelength bin is the mean of the flux in the old pixels that overlap the new bin, weighted by the extent of overlap. The error arrays associated with the resampled spectra are determined using a weighting method similar to the flux rebinning. The resampled fluxes are calculated using

$$
F_{r}=\frac{\sum_{i} F_{i}\left(\delta \lambda_{i}^{\prime}\right)}{\sum_{i}\left(\delta \lambda_{i}^{\prime}\right)},
$$

and their errors are the same as presented in Telfer et al. (2002). Here, $F_{r}$ is the flux of the rebinned pixel, and $F_{i}$ are the fluxes of pixels in the original spectrum. The widths $\left(\delta \lambda_{i}\right)$ are the sizes of the original wavelength bins, and the $\left(\delta \lambda_{i}^{\prime}\right)$ are the overlap of the old pixels with the new bin. Our initial composite spectrum used the bootstrap technique from Telfer et al. (2002) to combine the resampled spectra. We began with the spectrum of HE 0238-1904, which at $z=0.631$ lies at the median redshift of our sample. We defined it as our first partially formed composite spectrum and renormalized for convenience. We then included the remaining spectra in sorted order, alternating between higher-redshift spectra and lower-redshift spectra, after normalizing the continuumlike regions of each spectrum to the continuum of the partially formed composite spectrum.
We then explored ways of improving the composite. Ideally we would normalize all the spectra in the sample to each other at one common continuum window; the wide range of redshifts precludes such an approach. Instead, we find the rest-frame continuum window common to the most spectra $(860-880 \AA$ is shared by 12 of 22 spectra). We designate the spectra that share this continuum window as Group 1 and normalize those spectra within that wavelength region. A partially formed composite spectrum is produced by calculating the mean flux in each $0.1 \AA$ bin and renormalizing for convenience. The remaining spectra are separated into two other groups: Group 2 contains AGN at smaller redshifts than Group 1, and Group 3 are AGN at larger redshifts. We compile the spectra of Groups 2 and 3 with the Group 1 composite in the following steps: (1) normalize to the partially formed composite; (2) calculate a new partially formed composite; (3) renormalize the new partially formed composite; (4) repeat until all spectra in Groups 2 and 3 are compiled in the final composite. We normalize the spectra of Groups 2 and 3 to the partially formed composite by finding the weighted-mean normalization constant from multiple continuum windows, calculated using Equation (4) of Telfer et al (2002). Because the Group 1 composite represents the central region of the final composite, the order of adding Group- 2 and Group- 3 spectra to the composite does not matter. In practice, we added Group 2 spectra in order of decreasing redshift and Group-3 spectra in order of increasing redshift. To prevent narrow absorption features from affecting the normalization 
within the continuum windows, we normalize using spline fits to the individual spectra and use a partially formed composite of the spline fits.

Our final AGN composite spectrum uses spline fits to the individual spectra rather than the raw data themselves. This has the advantage of interpolating over narrow absorption features, which would otherwise complicate the coaddition of higher-redshift spectra. We did not include the restored continuum data from SBS $1108+560$ at $\lambda<770 \AA$, owing to the low $\mathrm{S} / \mathrm{N}$ from a strong LLS. We fit continua to each of the data sets, using a semiautomated line identification and spline-fitting technique as follows. First, the spectra are split into 5-10 $\AA$ segments. Continuum pixels within each segment are identified as those for which the $\mathrm{S} / \mathrm{N}$ (per pixel flux/error) vector is less than $1.5 \sigma$ below the $\mathrm{S} / \mathrm{N}$ in the segment. Thus, absorption lines are excluded, as are regions of increased noise. The process is iterated until minimal change occurs between one iteration and the next. The continuum pixels in a particular bin are then set, and the median continuum flux node is recorded. A spline function is fitted between continuum nodes. We check the continuum fits manually, and the continuum region identifications are adjusted as needed. The continuum identification and spline-fitting processes work reasonably well for smoothly varying data, but they were augmented with piecewise-continuous Legendre polynomial fits in a few cases. In particular, spline fits perform poorly in regions of sharp spectral curvature, such as the Galactic Ly $\alpha$ trough and at the peaks of cuspy emission lines. More details on the process are given elsewhere (C. W. Danforth et al. 2012, in preparation).

We now discuss sources of random and systematic uncertainty in the composite spectral indices. In our process, we fit two power laws to the spline composite spectrum, $\alpha_{\mathrm{FUV}}$ and $\alpha_{\mathrm{EUV}}$, and match them at a break wavelength $\lambda \approx 1000 \pm 50 \AA$. Although this break is apparent in the composite, its presence is less clear in the individual spectra. Because of the AGN redshift distribution of our sample $(z<0.16$ and $z>0.45)$ the rest-frame spectra lie either above or below the $1000 \AA$ break. To quantify the uncertainty in the fitting of the composite spectrum, we explore sources of uncertainty described by Scott et al. (2004), including the effects of cosmic variance in the shape of AGN SEDs, Galactic extinction, and formal statistical fitting errors. We do not include the effects of intrinsic absorbers, Ly $\alpha$ forest, or interstellar medium; these absorption lines are easily removed. The largest source of uncertainty comes from the natural variation in the slope of the contributing spectra. We estimate this uncertainty by selecting 1000 bootstrap samples with replacement from our sample of 22 AGN spectra. The standard deviations of the resulting spectral slope distributions are $\sigma=0.21$ for $\alpha_{\mathrm{EUV}}$ and $\sigma=0.14$ for $\alpha_{\mathrm{FUV}}$. These turn out to be the dominant sources of uncertainty.

We investigated uncertainties that arise from UV extinction corrections from two quantities: color excess $E(B-V)$ and ratio of total to selective extinction $R_{V}$. We alter the measured $E(B-V)$ by $\pm 16 \%(1 \sigma)$ as reported by Schlegel et al. (1998). We deredden the individual spectra with $E(B-V)$ multiplied by 1.16 or 0.84 , compile the spectra into a composite, and fit the con-
TABLE 2

Lyman Limit and Partial Limit Systems

\begin{tabular}{|c|c|c|c|}
\hline AGN Target & $\begin{array}{c}z_{\mathrm{LLS}^{\mathrm{a}}} \\
\text { (redshift) }\end{array}$ & $\begin{array}{c}\log \mathrm{N}_{\mathrm{HI}^{\mathrm{a}}} \\
\left(N_{\mathrm{HI}} \text { in cm }\right.\end{array}$ & $\begin{array}{c}b^{\mathrm{a}} \\
\left(\mathrm{km} \mathrm{s}^{-1}\right)\end{array}$ \\
\hline \multirow[t]{2}{*}{ HE 0439-5254 } & 0.61504 & 16.15 & 32 \\
\hline & 0.61565 & 15.6 & \\
\hline \multirow[t]{3}{*}{ SBS $1122+594$} & $\begin{array}{l}0.55743 \\
0.55810\end{array}$ & $\begin{array}{l}15.9 \\
16.25\end{array}$ & $\begin{array}{l}22 \\
15\end{array}$ \\
\hline & 0.5584 & 15.9 & 20 \\
\hline & 0.67822 & 16.3 & 18 \\
\hline \multirow[t]{2}{*}{ SBS $1108+560$} & 0.463 & 15.8 & ter \\
\hline & 0.46335 & 17.78 & 20 \\
\hline FBQS0751+2919 & 0.82915 & 16.1 & 30 \\
\hline \multirow{3}{*}{ PG $1407+265$} & 0.57497 & 15.6 & 28 \\
\hline & 0.59954 & 15.9 & 20 \\
\hline & 0.6827 & 16.35 & 33 \\
\hline \multirow[t]{2}{*}{ PG $1206+459$} & 0.92707 & 16.4 & $\cdots$ \\
\hline & 0.9276 & 17.1 & 30 \\
\hline \multirow[t]{2}{*}{ PG $1338+416$} & 0.68615 & 16.7 & 20 \\
\hline & 0.68645 & 15.5 & $\cdots$ \\
\hline Q $0232-042$ & 0.7389 & 16.8 & 35 \\
\hline
\end{tabular}

a For these 8 AGN sight lines, we found 17 Lyman limit or partial Lyman limit systems. We list their redshifts, H I column densities, and doppler parameters $(b)$ derived by fitting Ly $\alpha$ and higher Lyman series absorbers.

tinua. We find that $\alpha_{\mathrm{EUV}}$ changes by $(+0.025,-0.021)$ while $\alpha_{\text {FUV }}$ changes by $(+0.045,-0.043)$. Next, we estimate the sensitivity to deviations from the canonical value $R_{V}=3.1$ by dereddening the individual spectra with $R_{V}=2.8$ and $R_{V}=4.0$ and compiling the spectra into composites. We find that $\alpha_{\mathrm{EUV}}$ changes by $(+0.017,-0.044)$ and $\alpha_{\mathrm{FUV}}$ by $(+0.026,-0.076)$. The formal statistical errors for the spectral indices are negligible $(<0.0015)$, owing to the high $\mathrm{S} / \mathrm{N}$ ratio of our composite spectra, and we do not include them in the final quoted uncertainties. We add the random uncertainties of cosmic variance with the systematic effects of correcting for extinction in quadrature and estimate the total uncertainties to be \pm 0.21 for $\alpha_{\mathrm{EUV}}$ and \pm 0.15 for $\alpha_{\mathrm{FUV}}$.

Obtaining a larger AGN sample will be helpful, particularly for the shorter wavelengths $(\lambda<900 \AA)$ for which only eight AGN contribute. In the future, we plan to create a composite AGN spectrum between 300 and 1800 $\AA$, using $H S T / \mathrm{COS}$ archival spectra of AGN with a variety of types and luminosities. The order-of-magnitude sensitivity increase offered by COS over previous spectrographs has increased the number of targets available for far-UV spectroscopy to over 270 AGN. These archival spectra will provide EUV coverage down to $500 \AA$ (with $\sim 40$ AGN) and to $912 \AA$ (with 100 AGN). For even shorter rest-frame wavelengths $(304-500 \AA)$ we can use the sample of quasars (Shull et al. 2010; Worseck et al. 2011; Syphers et al. 2011) observed to study the He II reionization epoch. These "He II quasars" are the rare subset of $z \sim 3$ quasars that are FUV-bright because of low hydrogen column density. There are now 31 such FUV-bright quasars observed with COS, of which 27 have flux extending down to $304 \AA$. Below this wavelength, intergalactic He II absorbs most or all of the flux. While most of these spectra have low $\mathrm{S} / \mathrm{N}$, when combined they will extend the composite EUV spectrum out to 3 ryd in energy. For comparison, Telfer et al. (2002) 
had only one quasar extending to $304 \AA$ and Scott et al. (2004) had none.

\section{RESULTS}

The results of our AGN spectral fits and UV/EUV composite spectra are displayed in Figures 2-7. Table 1 provides information about the 22 individual AGN, the power-law fits to their $H S T / \mathrm{COS}$ spectra, and their band-luminosities, $\lambda L_{\lambda}$, at $1100 \AA$, given by

$$
\begin{aligned}
\lambda L_{\lambda} & =\left(1.32 \times 10^{43} \mathrm{erg} \mathrm{s}^{-1}\right)\left[\frac{d_{L}}{100 \mathrm{Mpc}}\right]^{2} \\
& \times\left[\frac{F_{\lambda}}{10^{-14} \mathrm{erg} \mathrm{cm}^{-2} \mathrm{~s}^{-1} \AA^{-1}}\right]\left[\frac{\lambda}{1100 \AA}\right] .
\end{aligned}
$$

Here, we converted flux, $F_{\lambda}$, to luminosity, $L_{\lambda}=4 \pi d_{L}^{2} F_{\lambda}$, using the luminosity distance, $d_{L}(z)$, computed for a flat $\Lambda \mathrm{CDM}$ universe with $H_{0}=70 \mathrm{~km} \mathrm{~s}^{-1} \mathrm{Mpc}^{-1}$ and density parameters $\Omega_{m}=0.275$ and $\Omega_{\Lambda}=0.725$ (Komatsu et al. 2011). Table 3 shows the AGN indices from composite spectra found from various surveys (1991-2004) compared to the current COS survey (2012). The median values of $\alpha_{\nu}$ from these surveys depend on the rest wavelengths sampled, since many quasar SEDs involve both power-law distributions and broad features ("big blue bump", "small UV bump") attributed to thermal emission from the accretion disk (Shields 1978) and to blends of Fe II emission (Wills et al. 1985). In general, the SEDs steepen from the optical and near-UV bands, where $F_{\nu} \propto \nu^{-0.3}$ or $\nu^{-0.4}$, to distributions $\nu^{-1.4}$ or $\nu^{-2.0}$ at shorter (FUV and EUV) wavelengths. The break in the COS composite occurs at $\sim 1000 \AA$, well shortward of Ly $\alpha(1216 \AA)$. In future $H S T / \mathrm{COS}$ archival studies, we intend to find AGN in the critical range $0.15<z<0.5$, whose rest-frame spectra probe the $1000 \AA$ break.

Figure 2 shows two outstanding examples of farUV spectra taken by COS, labeling prominent emission lines in the low-redshift Seyfert 1.5 galaxies, Mrk 817 and Mrk 1513. According to the classification scheme proposed by Richards et al. (2011), Mrk 817 is a "wind-dominated AGN" with weak Ly $\alpha$, Si IV, and He II emission lines and blue-shifted $\mathrm{N} \mathrm{V}$ and $\mathrm{O}$ IV]. Mrk 1513 is a narrow-line Seyfert galaxy, exhibiting a "disk-dominated" spectrum with prominent emission lines (Ly $\alpha$, C IV, Si IV). Figures 3-5 show individual spectra of all 22 AGN in our sample. Table 4 lists the detected or expected emission lines and their rest wavelengths. Most of the low- and intermediate-redshift spectra show the prominent ultraviolet emission lines seen in optical spectra of higher-redshift QSOs: Ly $\alpha$, C IV, N V, O VI, Si IV+O IV]. The weaker lines (centroids of blends) include O I 1303 + Si II 1307, C II 1335, He II 1640, O III] 1665 , and many others at shorter wavelengths. A number of weak lines show up in the composite spectrum, including Fe III 1123, N II 1085, N III 989-991, N III] 1750, N IV] 1486, and two emission lines of C III (1175 and $977 \AA$ ). The semi-forbidden line of C III] $\lambda 1909$ lies outside the range of the COS G160M grating.

Figure 6 shows the composite UV/EUV spectrum for the full sample of 22 AGN, extending from $550 \AA$ to $1750 \AA$ in the AGN rest frame. Because these AGN range in redshift from $z_{\min }=0.026$ to $z_{\max }=1.44$, wavelengths in the composite include contributions from a limited number of spectra, typically $3-12$, as shown in the bottom panel of Figure 6 . These effects are further illustrated in Figure 7, with composite spectra for subsets of seven low-redshift AGN and eight high-redshift AGN. These subsets probe the FUV and EUV portions, respectively, in the AGN rest frame. In the EUV range, we see a number of broad emission lines (Table 4) from higher ionization states of oxygen, nitrogen, and neon. The most prominent EUV emission-line blends are O II $(834 \AA)$ and O III $(833 \AA)$, the Ne VIII doublet $(770 \AA$ and $780 \AA)$, and Ne V (569 $\AA)$. We also detect weaker lines of N III (685 $\AA)$, N IV (765 $\AA$ ), and a range of high ionization states of oxygen, including O III (703 $\AA$ ), O IV $(788,608,554 \AA)$, and O V (630 $)$. Some of the difference in the FUSE composite index (Scott et al. 2004) may come from incorrectly fitting the continuum through these EUV emission lines. These lines from high ionization states provide important diagnostics of BELR physical conditions, particularly multiple ions from the same element such as Ne V and Ne VIII, or O III, O IV, O V, and $\mathrm{O}$ VI.

Figure 8 looks for possible dependences of the spectral indices on AGN redshift and luminosity, $\lambda L_{\lambda}$ (at $1100 \AA$ ). The symbols correspond to the redshift subgroups (Table 1), which have mean values, $\left\langle\log \lambda L_{\lambda}\right\rangle=$ $44.44,46.08$, and 46.31 for groups at $\langle z\rangle=0.070,0.565$, and 1.07 , respectively. We find a weak correlation of individual spectral indices with each. Both likely reflect the same trend, in which higher-luminosity, higher-redshift AGN sample the rest-frame continuum at progressively shorter wavelengths. We see a steepening of the flux distribution, $F_{\nu}$, which corresponds to a flattening in $F_{\lambda}$. Because different redshift ranges sample the SED on both sides of the $1000 \AA$ break, samples of AGN at higher redshifts are biased toward softer spectra. These correlations will be explored further with larger samples from COS archival data. We will also explore possible dependence on black hole mass. Three of the AGN in our survey have black hole mass estimates obtained from observed C IV line widths and continuum luminosities at $1350 \AA$ (Vestergaard \& Peterson 2006). These mass estimates are: $\log \left(M_{\mathrm{BH}} / M_{\odot}\right)=7.41,7.93$, and 8.85 for Mrk 335, Mrk 817, and Mrk 876, respectively, based on UV data from $I U E$ and $H S T / F O S$. Our new spectra will provide accurate C IV line widths and 1300 A luminosities for the first seven AGN in Table 1, at $z<0.155$. At higher redshifts, the C IV emission line shifts out of the COS/G160M band. The correlation between $M_{\mathrm{BH}}$ and C IV line width is controversial (Netzer et al. 2007; Assef et al. 2011) owing to scatter in the relation and likely changes with AGN luminosity (Shen et al. 2008). We will further analyze the AGN in our survey for correlations with C IV and other emission lines. 

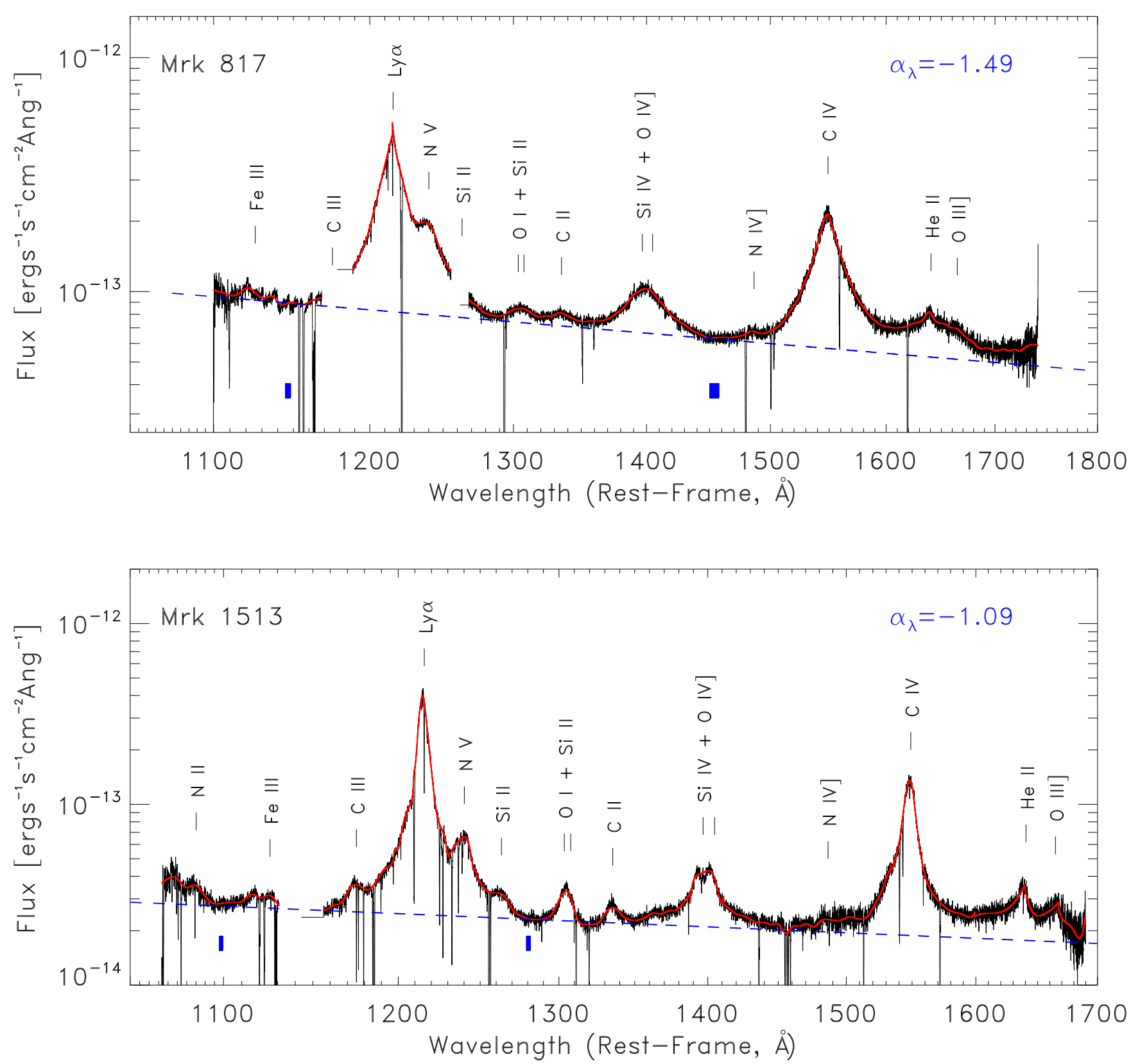

FIG. 2.- COS spectra of Mrk 817 and Mrk 1513, binned to a COS resolution element $(\sim 0.08 \AA)$ by smoothing over 7 pixels and binning by 3 pixels. Red line is spline fit. We label prominent emission lines (Table 4), line-free continua (blue boxes), and excise gaps from Galactic Ly $\alpha$ absorption. Fitted far-UV spectral indices, $\alpha_{\lambda}$, are in top right corners. 


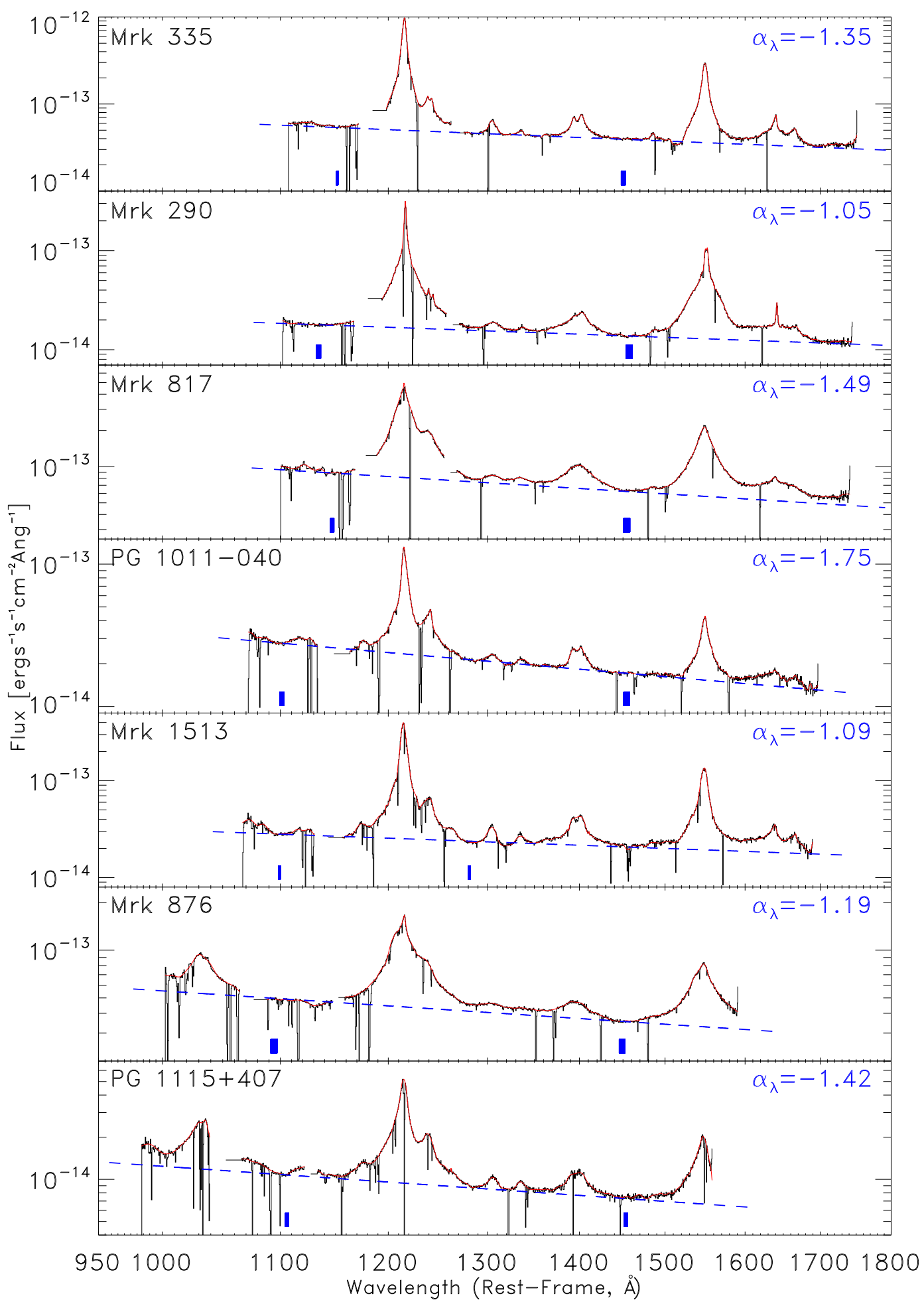

FIG. 3.- First of three plots that show de-reddened COS spectra and fitted spectral indices $\alpha_{\lambda}$ (top right corners) for seven low-redshift AGN $(0.026 \leq z \leq 0.154)$ in the survey. Spectra are smoothed over 7 pixels and binned to 52 pixels $(0.5 \AA)$. Red line is a spline fit. 


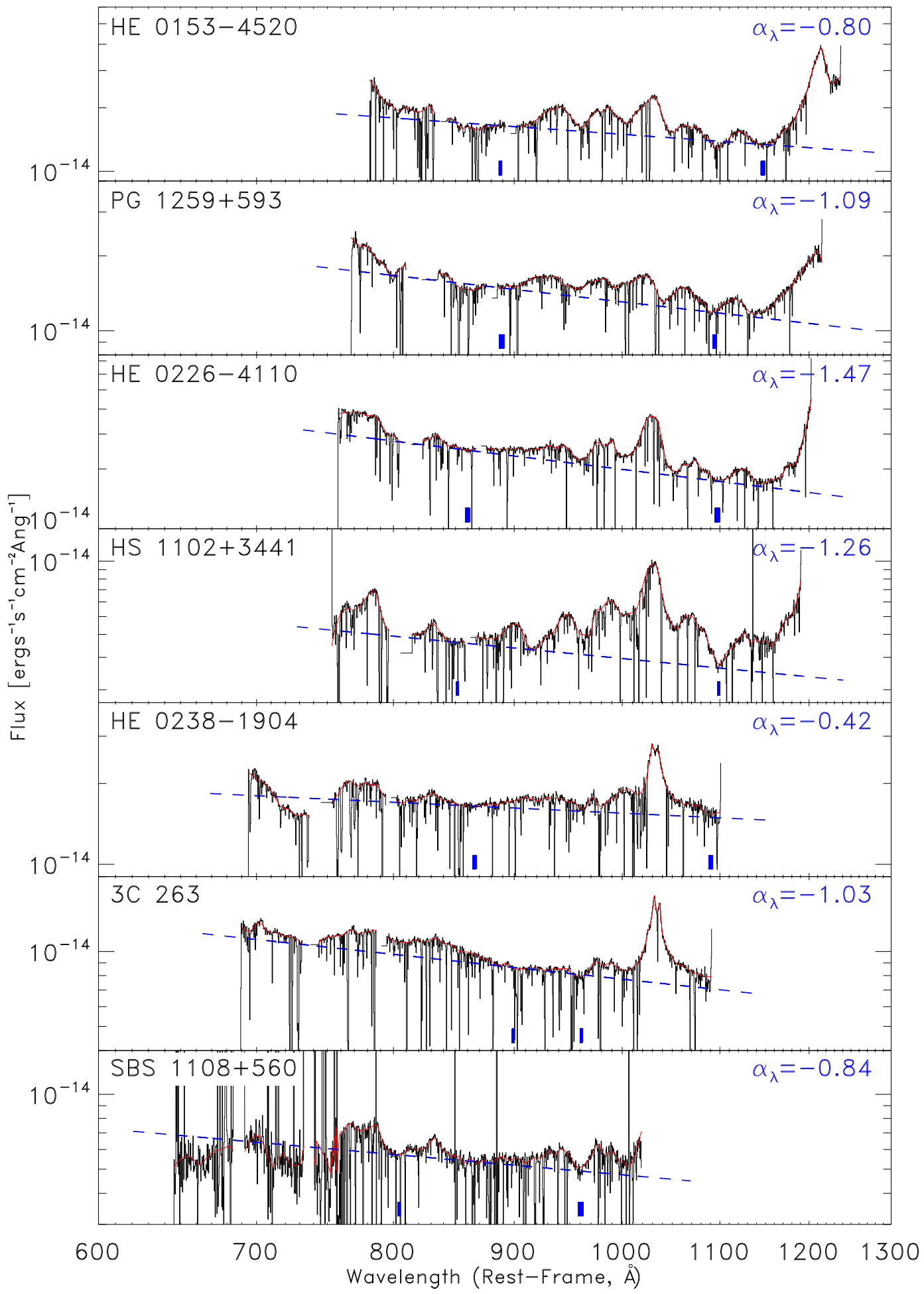

FIG. 4. - Same as Figure 3, showing de-reddened COS spectra for seven intermediate-redshift AGN $(0.45 \leq z \leq 0.77)$ in survey. Restored continuum at $\lambda<755 \AA$ (AGN rest frame) toward SBS 1108+560 is unreliable, owing to the presence of a strong LLS at $z=0.46445$. 


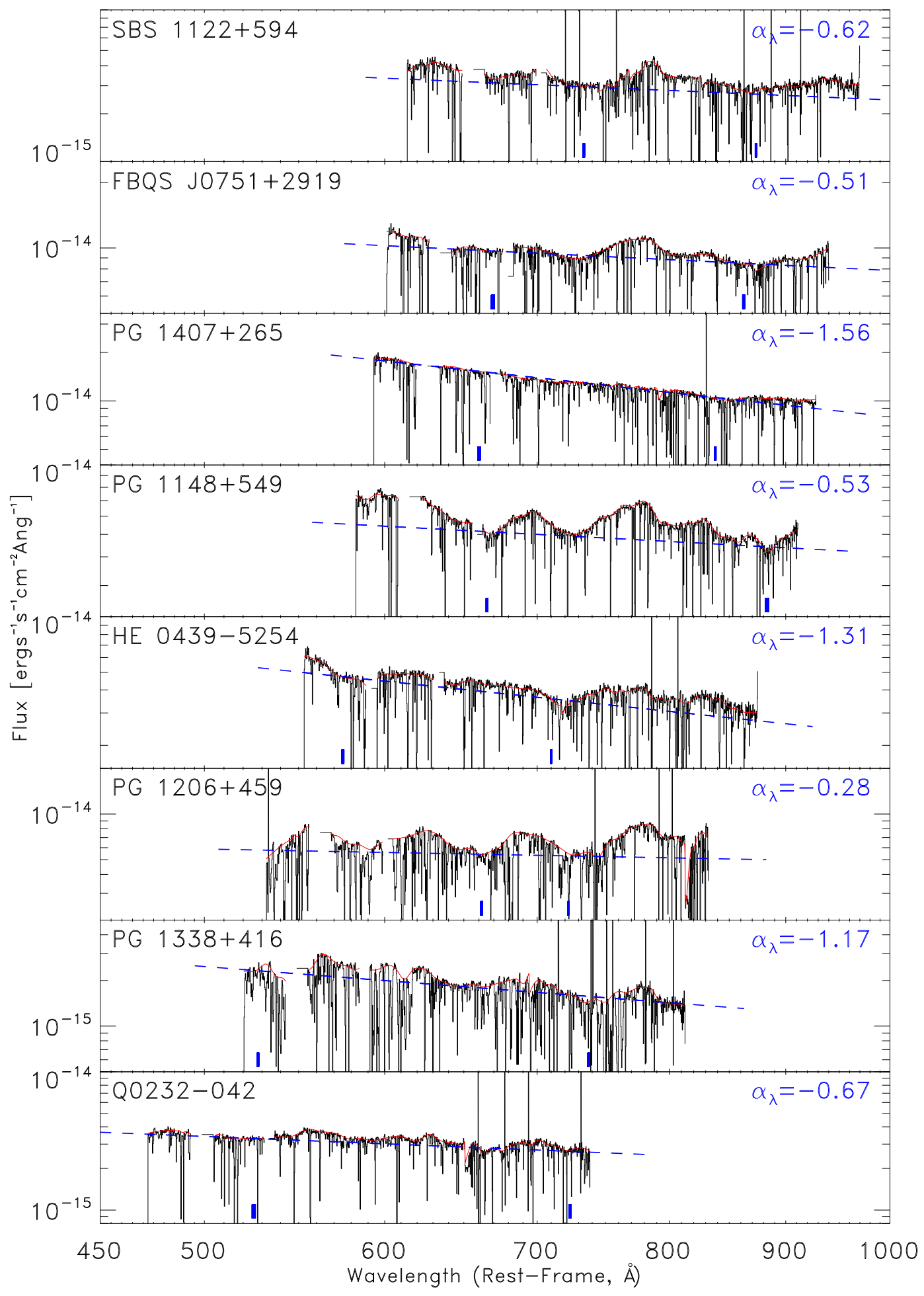

FIG. 5.- Same as Figure 3, showing de-reddened COS spectra for eight high-redshift AGN $(0.85 \leq z \leq 1.44)$ in the survey. 


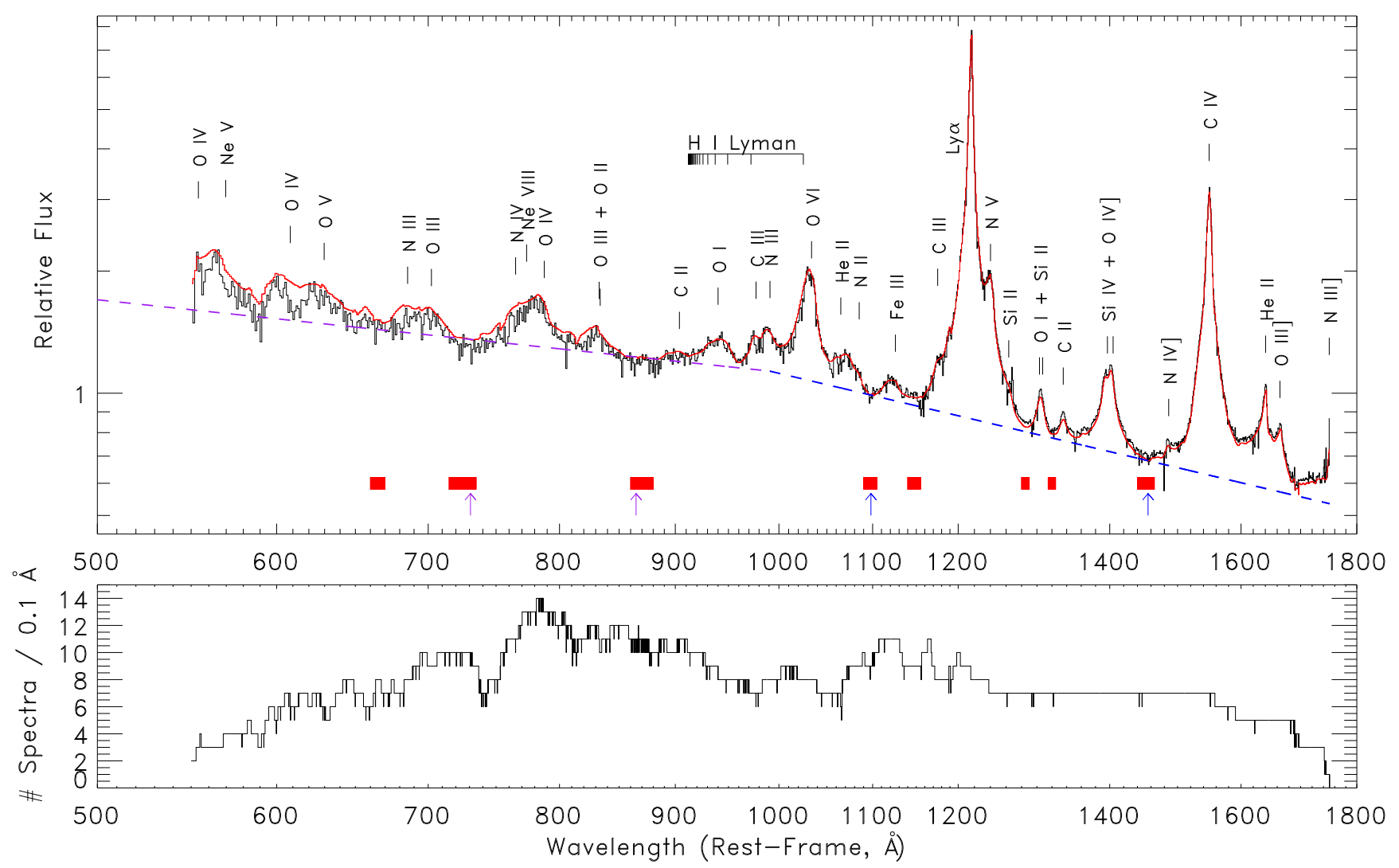

FIG. 6.- Composite $H S T / \mathrm{COS}$ spectrum of all $22 \mathrm{AGN}$ at $0.026 \leq z_{\mathrm{em}} \leq 1.44$, resampled to $0.1 \AA$, plotted in $1 \AA$ bins, normalized to 1 at $1100 \AA$, and showing array of broad FUV and EUV emission lines $\left(550 \AA<\lambda_{\text {rest }}<1220 \AA\right)$ atop a power-law continuum. Eight continuum windows are shown as small red boxes. Composite data are shown in black, and red curve is composite of individual spline fits. Lower panel shows number of AGN targets contributing to composite spectrum vs. wavelength. We can distinguish regions of true continuum (noted by small arrows) from emission lines and "pseudo-continuum" composed of blended emission lines. The power-law continuum exhibits a break at $\lambda \approx 1000 \pm 50 \AA$, with a flatter (softer) spectrum at EUV wavelengths. The frequency distribution, $F_{\nu} \propto \nu^{\alpha_{\nu}}$, has indices $\alpha_{\nu}=-1.41 \pm 0.21$ (EUV, $\left.\lambda<1000 \AA\right)$ and $\alpha_{\nu}=-0.68 \pm 0.14(\mathrm{FUV}, \lambda>1200 \AA)$. 

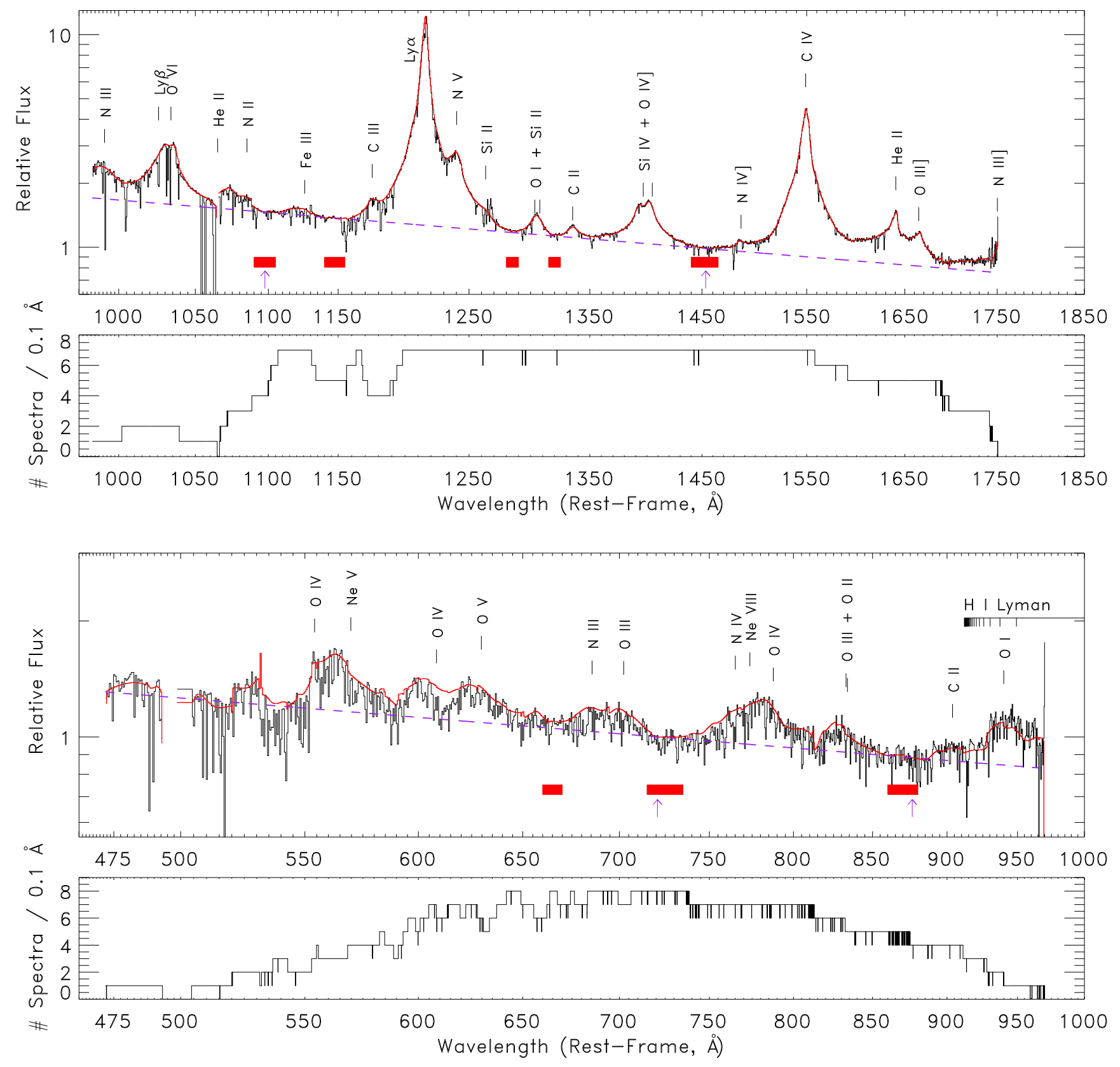

FIG. 7.- (Top) Composite COS spectrum of seven low-redshift AGN at $z_{\mathrm{em}} \leq 0.154$, normalized at $1450 \AA$, resampled to $0.1 \AA$ and plotted in $0.5 \AA$ bins. Continuum windows are shown as small red boxes, and red curve is our spline fit. The FUV power-law continuum $(\lambda>1200 \AA)$ has index $\alpha_{\nu}=-0.58 \pm 0.2$, similar to the overall (22-QSO) composite value of $-0.68 \pm 0.14$ shown in Figure 6 . (Bottom) Composite COS spectrum of eight high-redshift AGN at $0.85<z_{\mathrm{em}}<1.44$. normalized at $733 \AA$. The EUV power-law continuum $(\lambda<1000 \AA)$ has index $\alpha_{\nu}=-1.37 \pm 0.20$, similar to the overall composite value of $-1.41 \pm 0.21$ in Figure 6. 

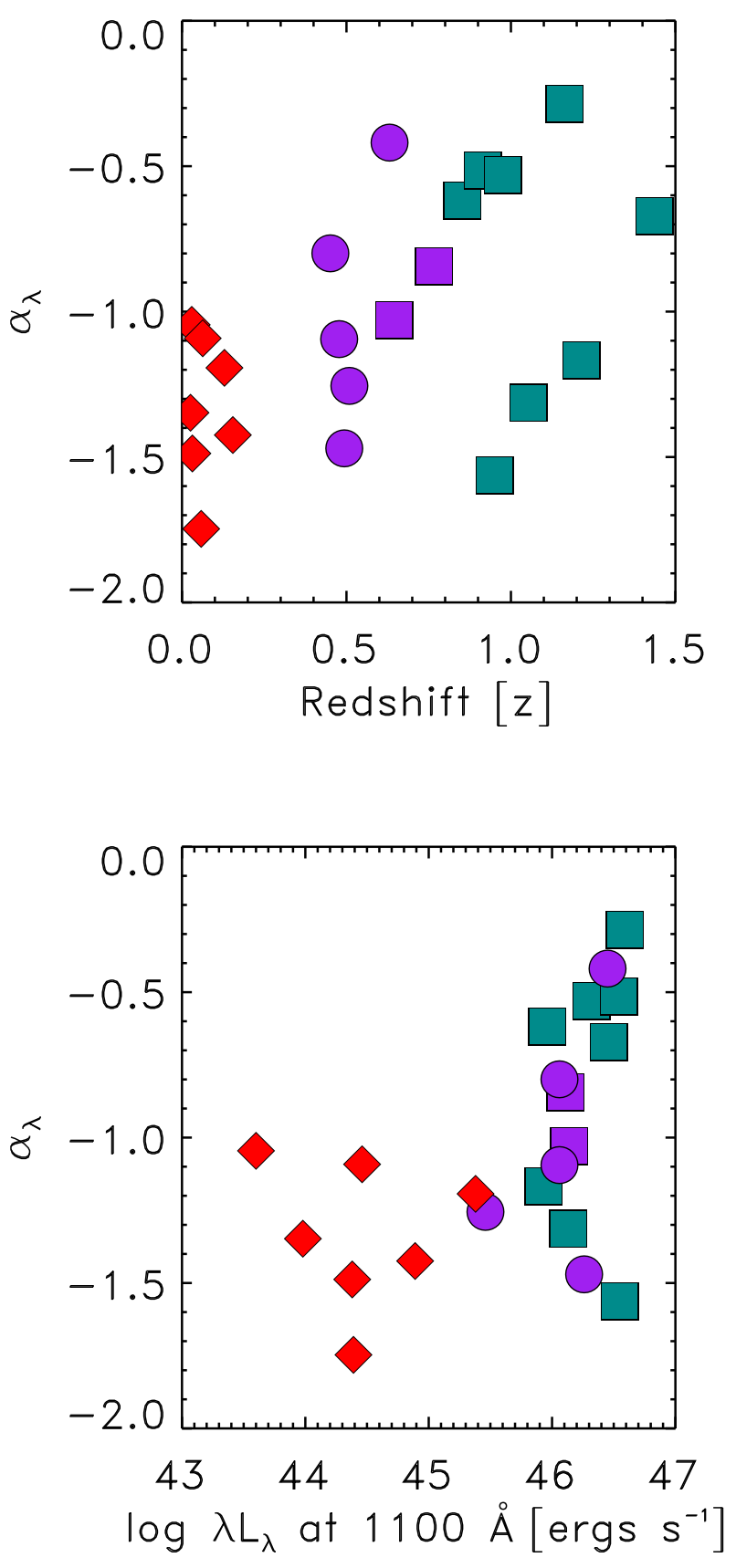

FIG. 8.- Correlations of fitted spectral indices, $\alpha_{\lambda}$, with AGN redshift (top) and $1100 \AA$ luminosity (bottom) for our 22 AGN. Colors correspond to the three redshift groups: red (seven low-redshift AGN in Figure 3); purple (seven intermediate-redshift AGN in Figure 4); turquoise (eight high-redshift AGN in Figure 5). Symbol shapes refer to the wavelength windows used to fit the continua: diamonds have windows longward of $1000 \AA$, squares have windows shortward of $1000 \AA$, and circles have windows that straddle $1000 \AA$, with one window (each) in EUV and FUV.

Although the statistical quality of COS data exceeds that of FUSE and IUE, we thought it prudent to compare the spectra produced by these three ultraviolet spectrographs. Figure 9 shows stitched spectra for four AGN, with combined data from COS, FUSE, and IUE. The FUSE and COS data were patched together at the following wavelengths: Mrk 290 (1141.3 ̊), Mrk 817 (1136.6 ̊), PG 1259+593 (1153.2 ̊), and 3C 363 (1136.6 $\AA$ ). The COS and IUE data were patched together at $1796.5 \AA$. For these four AGN, the spectral indices fitted to $H S T / C O S$ data provide reasonable fits to the data, both at shorter wavelengths (FUSE) and longer wavelengths (IUE).

Figure 10 compares our COS composite spectrum with the AGN composites found from the Sloan Digital Sky Survey (SDSS) using new redshifts from Hewett \& Wild (2010). The SDSS composites were most recently presented by Richards et al. (2011) in four sub-groups, defined by their line-to-continuum ratios. The COS composite exhibits stronger emission lines than those of any of the SDSS sub-groups, suggesting that many of our AGN have "disk-type" spectra defined by Richards et al. (2011) and more likely to be radio-loud with hard ionizing spectra. However, only 2 of the 22 AGN (3C 263 and Q0232-042) are radio-loud, as measured by the ratio of fluxes at $5 \mathrm{GHz}$ and optical (V band). This $10 \%$ fraction is consistent with the QSO radio-loud fraction.

We conclude this section with a few general results from the composite spectra. We see no trace of the Lyman continuum edge $(912 \AA)$ in the rest frame of the composite spectrum, with an optical-depth limit $\tau_{\mathrm{HI}}<0.03$. Possible explanations of the lack of a Lyman edge in accretion-disk atmosphere models (Davis et al. 2007 ) include hot, highly ionized disk photospheres and possible temperature inversions arising from external irradiation (Siemiginowska et al. 1995). We also see no obvious emission line from He II $\lambda 584$, which could be present in the broad emission-line region. Because this line is formed deep inside BELR clouds, it will scatter resonantly and be destroyed by $\mathrm{H}$ I Lyman continuum absorption. However, only four AGN contribute to the composite at $584 \AA$, and any weak He I line would appear in the broad red wings of O IV $\lambda 554$ and $\mathrm{Ne} V$ $\lambda 569$. We also see no "Lyman Valley" (Møller \& Jakobsen 1990), an extremely broad absorption feature centered at $\lambda \sim 650 \AA$ that may arise from the cumulative Lyman continuum edges of translucent $\mathrm{H}$ I absorbers at moderate redshift. The expected effect is small $(\sim 5 \%)$ at $z<1$. 

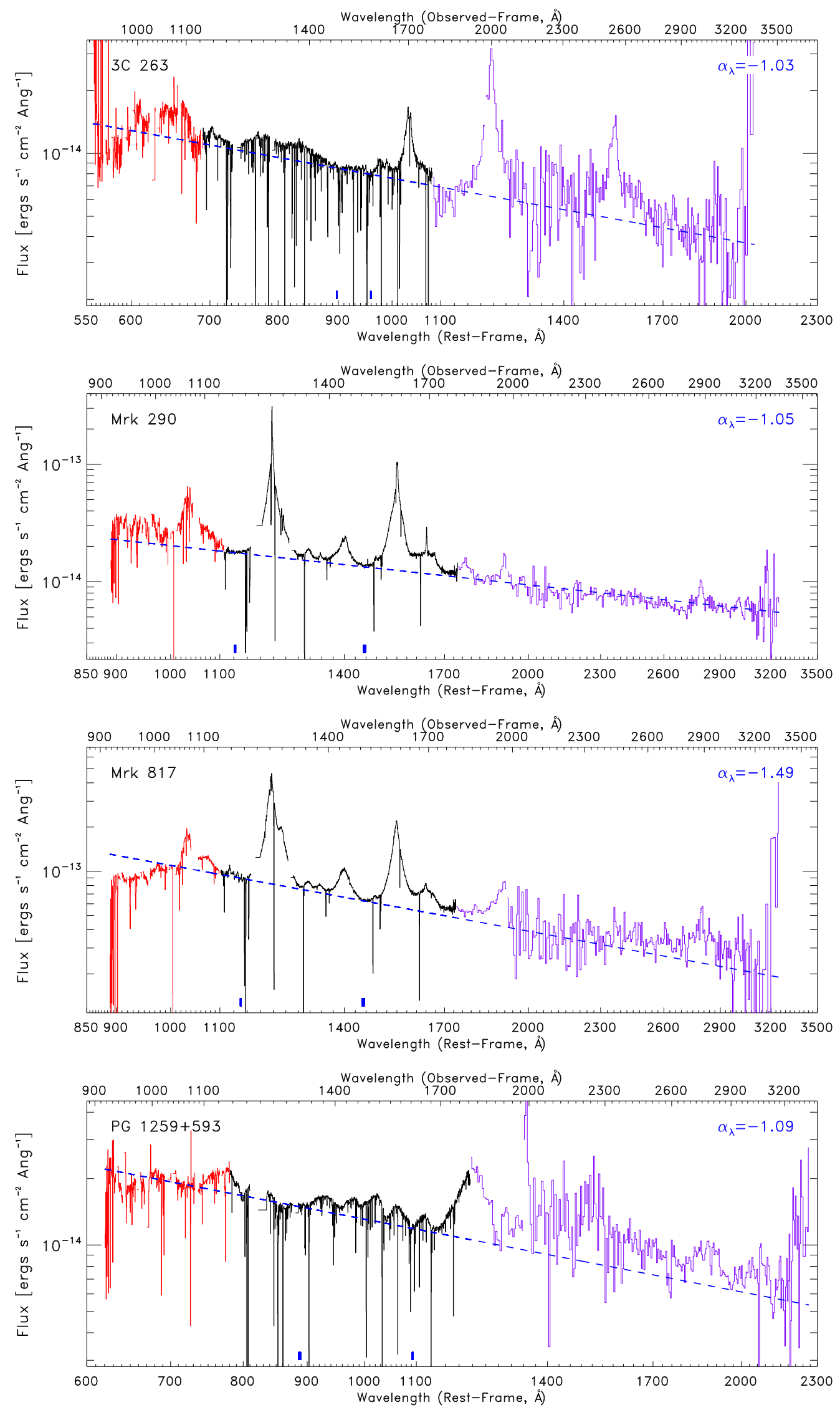

FIG. 9. - Stitched spectra of 3C 263, Mrk 290, Mrk 817, PG 1259+593 (top to bottom) combining spectral data from FUSE (shortest wavelengths), HST/COS (middle range), and IUE (longest wavelengths). Transitions are apparent from line texture. We excised H I and O I airglow lines. Data from FUSE and COS are binned to $0.5 \AA$, and IUE data to $4.5 \AA$ (short-wavelength) or $5.0 \AA$ (long wavelength). The fitted spectral indices, $\alpha_{\lambda}$, are those from COS alone (Table 1), but they also provide reasonable fits to the FUSE and IUE portions. 


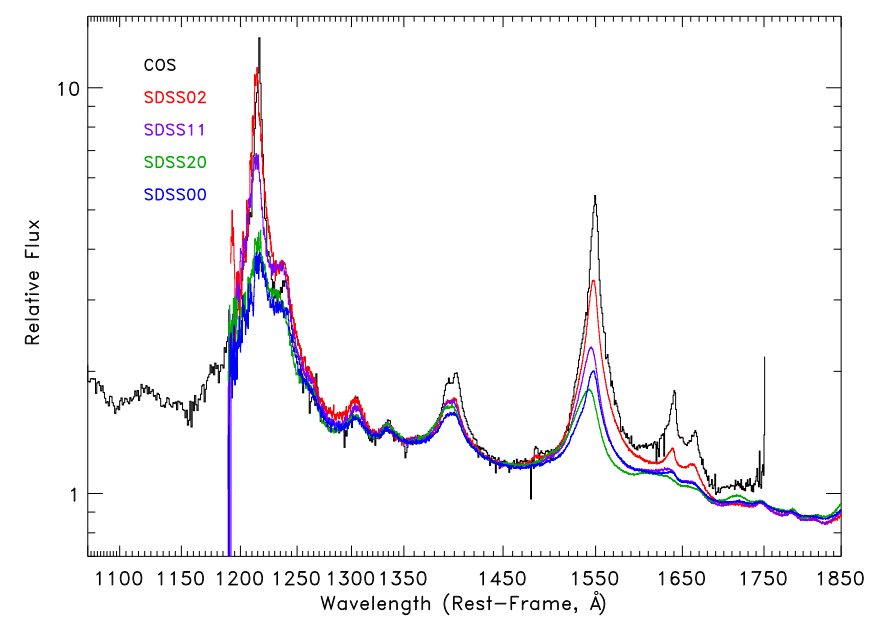

FIG. 10.- Overplot of COS composite spectrum with four composite spectra from SDSS (Richards et al. 2011; Hewett \& Wild 2010). Four SDSS colors refer to AGN classes with progressively stronger emission lines, referred to as "disk-type" (strong emission lines, hard spectra) and "wind-type" (weak emission lines, softer spectra). The hard spectra exhibit strong He II $\lambda 1640$ emission lines. Our 22 AGN with COS are primarily "disk type" with strong emission lines, particularly Ly $\alpha$, C IV, He II, O III], Si IV/O IV].

\section{DISCUSSION AND CONCLUSIONS}

We now summarize the results and implications of our survey of AGN spectral distributions in the rest-frame FUV and EUV, using spectra of 22 AGN studied by $H S T / C O S$. We achieved the primary goal of our initial study, which was to investigate the difference between the markedly different spectral indices, $\alpha_{\nu}$, obtained by earlier surveys with $H S T$ /FOS (Telfer et al. 2002) and FUSE (Scott et al. 2004). Our major results are:

1. Two previous ultraviolet surveys of the spectra of AGN gave conflicting results for their composite rest-frame LyC spectra taken by $H S T / F O S$ and FUSE. High-S/N spectra of 22 AGN taken with HST/COS G130M and G160M gratings give a composite EUV spectral index, $\alpha_{\nu}=-1.41 \pm 0.21$, in reasonable agreement with the HST/FOS value, $\alpha_{\nu}=-1.57 \pm 0.17$ (Telfer et al. 2002) for radioquiet AGN.

2. For wavelengths $\lambda>1200 \AA$, previous AGN surveys found spectral indices $\alpha_{\nu}$ between -0.3 and -0.6 , steepening to $\alpha_{\nu} \approx-1.6$ at $\lambda<1000 \AA$. The composite indices depend on sample size and the range of QSO luminosity and redshift. The continuum must be carefully fitted beneath strong EUV emission lines and is sensitive to how one combines QSOs with a wide range of individual spectral indices.

3. The 22 AGN exhibit a wide range of individual AGN spectral indices at $\lambda<1100 \AA$, ranging from $\alpha_{\nu}=-0.25$ (for PG 1011-040 with a flat, hard spectrum) to $\alpha_{\nu}=-1.75$ (for PG 1206+459 with a steep, soft spectrum). Most spectra show a break at rest wavelength $\lambda \approx 1000 \AA$, with a steepening of the flux distribution, $F_{\nu} \propto \nu^{\alpha_{\nu}}$, at higher frequencies and $\lambda<1000 \AA$.
4. We see no Lyman edge at $912 \AA$ or He I $\lambda 584$ emission line in the AGN composite, which has implications for models of accretion-disk atmospheres and AGN emission-line regions. Our COS AGN composite has stronger emission lines (Ly $\alpha, \mathrm{C}$ IV, Si IV, He II) than the SDSS composites (Richards et al. 2011), suggesting that these AGN are of "disk type", with hard ionizing spectra and strong He II $\lambda 1640$ emission.

We thank Brian Keeney, Stéphane Béland, and the COS/GTO team for help on the calibration and verification of early COS data, and Gordon Richards and Paul Hewett for sharing their SDSS composite spectra. Brad Peterson and Paulina Lira provided advice on the use of C IV as a black-hole mass diagnostic. John Stocke, Shane Davis, and James Green provided helpful comments on this project. This research was supported by NASA grants NNX08-AC14G and NAS5-98043 and the Astrophysical Theory Program (NNX07-AG77G from NASA and AST07-07474 from NSF) at the University of Colorado Boulder. 


\section{REFERENCES}

Aguirre, A., Schaye, J., Kim, T.-S., et al. 2004, ApJ, 602, 38

Assef, R. J., Denney, K. D., Kochanek, C. S., et al. 2011, ApJ, 742, 93

Brotherton, M. S., Tran H. D., Becker R. H., et al. 2001, ApJ, 546, 775

Carballo, R., Gonzalez-Serrano, J., Benn, C., Sanchez, S., \& Vigotti, M. 1999, MNRAS, 306, 137

Cardelli, J. A., Clayton, G. C., \& Mathis, J. S. 1989, ApJ, 345, 245

Clavel, J., Reichert, G. A., Alloin, D., et al. 1991, ApJ, 366, 64

Crenshaw, D. M., Kraemer, S. B., \& George, I. M. 2003, ARA\&A, 41,117

Crenshaw, D. M., Rodriguez-Pascual, Penton, S. V., et al. 1996, ApJ, 470, 322

Danforth, C. W., Keeney, B. A., Stocke, J. T., Shull, J. M., \& Yao, Y. 2010, ApJ, 720, 976

Danforth, C. W., \& Shull, J. M. 2008, ApJ, 679, 194

Davis, S. W., Woo, J.-H., \& Blaes, O. M. 2007, ApJ, 688, 682

Edelson, R. A., Alexander, T., Crenshaw, D. M., et al. 1996, ApJ, 470,364

Edelson, R. A., Koratkar, A., Nandra, P., et al. 2000, ApJ, 534, 180

Fan, X., Narayanan, V., Lupton, R. H., et al. 2001, AJ, 122, 2833

Fardal, M. A., Giroux, M. L., \& Shull, J. M. 1998, AJ, 115, 2206

Fechner, C., Reimers, D., Kriss, G. A., et al. 2006, A\&A, 455, 91

Fitzpatrick, E. L. 1999, PASP, 111, 63

Francis, P. J., Hewett, P. C., Foltz, C. B., et al. 1991, ApJ, 373 465

Green, J. C., Froning, C., Osterman, S., et al. 2012, ApJ, 744, 60

Haardt, F., \& Madau, P. 2001, in Clusters of Galaxies and the High Redshift Universe Observed in X-rays, ed. D. M. Neumann \& J. T. V. Tran, (Saclay: CEA), 64 (arXiv:astroph/0106018)

Haardt, F., \& Madau, P. 2012, ApJ, 746, 125

Hasinger, G. 1994, in Frontiers of Space and Ground-Based Astronomy: The Astrophysics of the $21^{\text {st }}$ Century, ed. W. Wamsteker, M. Longair, \& Y. Kondo, ASSL, Vol. 187; Dordrecht: Kluwer), 381

Hasinger, G., Burg, R., Giacconi, R., et al. 1993, A\&A, 275, 1

Hewett, P. C., \& Wild, V. 2010, MNRAS, 404, 2302

Komatsu, E., Smith, K. M., Dunkley, J., et al. 2011, ApJS, 192. 18

Krolik, J. H., \& Kallman, T. 1988, ApJ, 324, 714

Markwardt, C. B. 2009, in ASP Conf. Ser. 411, Astronomical Data Analysis Software and Systems XVIII, ed. D. Bohlender, D. Durand, \& P. Dowler (San Francisco, CA: ASP), 251
Møller, P., \& Jakobsen, P. 1990, A\&A, 228, 299

Morton, D. C. 2003, ApJS, 149, 205

Netzer, H., Lira, P., Trakhtenbrot, B., Shemmer, O., \& Cury, I. 2007, ApJ, 671, 1256

Osterman, S., Green, J., Froning, C., et al. 2011, Ap\&SS, 335, 257

Reichert, G. A., Rodriguez-Pascual, P. M., Alloin, D., et al. 1994, ApJ, 425, 582

Richards, G. T., Kruczek, N. E., Gallagher, S. C., et al. 2011, AJ, 141, 167

Rodriguez-Pascual, P. M., Alloin, D., Clavel, J., et al. 1997, ApJS, 110,9

Schaye, J., Aguirre, A., Kim, T., et al. 2003, ApJ, 596, 768

Schlegel, D. J., Finkbeiner, D. P., \& Davis, M. 1998, ApJ, 500, 525

Schneider, D. P., Schmidt, M., \& Gunn, J. E. 1991, AJ, 101, 2004

Scott, J. E., Kriss, G. A., Brotherton, M., et al. 2004, ApJ, 615, 135

Shen, Y., Greene, J. E., Strauss, M. A., Richards, G. T., \& Schneider, D. P. 2008, ApJ, 680, 169

Shields, G. A. 1978, Nature, 272, 706

Shull, J. M., France, K., Danforth, C. W., Smith, B. D., \& Tumlinson, J. 2010, ApJ, 722, 1312

Shull, J. M., Roberts, D., Giroux, M. L., Penton, S. V., \& Fardal, M. A. 1999, AJ, 118, 1450

Shull, J. M., Tumlinson, J., Giroux, M. L., Kriss, G. A., \& Reimers, D. 2004, ApJ, 600, 570

Siemiginowska, A., Kuhn, O., Elvis, M., et al. 1995, ApJ, 454, 77 Syphers, D., Anderson, S. F., Zheng, W., et al. 2011, ApJ, 726, 111 Telfer, R., Zheng, W., Kriss, G. A., \& Davidsen, A. F. 2002, ApJ, 656,773

Vanden Berk, D. E., Richards, G. T., Bauer, A. et al. 2001, AJ, 122,549

Verner, D. A., Barthel, P. D., \& Tytler, D. 1994, A\&AS, 108, 287

Vestergaard, M., \& Peterson, B. M. 2006, ApJ, 641, 689

Wills, B. J., Netzer, H., \& Wills, D. 1985, ApJ, 188, 94

Worseck, G., Prochaska, J. X., McQuinn, M., et al. 2011, ApJ, 733, L24

Zheng, W., Kriss, G. A., Telfer, R. C., Grimes, J. P., \& Davidsen, A. F. 1997, ApJ, 475, 469 
TABLE 3

AGN Composite Spectral-Index Fits (FUV and EUV)

\begin{tabular}{|c|c|c|c|c|}
\hline Survey Reference $^{\mathrm{a}}$ & $N_{\mathrm{QSO}}{ }^{\mathrm{a}}$ & $\alpha_{\nu}{ }^{\mathrm{a}}$ & $\lambda$-range ${ }^{\mathrm{a}}$ & Survey Characteristics ${ }^{\mathrm{a}}$ \\
\hline Francis et al. (1991) & 718 & -0.32 & $1450-5050 \AA$ & LBQS (Large Bright Quasar Survey) \\
\hline Schneider et al. (1991) & 30 & $-0.92 \pm 0.05$ & $1270-1600 \AA$ & CCD grism survey $(z>3.1)$ \\
\hline Carballo et al. (1999) & 48 & $-0.87 \pm 0.20$ & $1300-4500 \AA$ & B3-VLA (radio) QSOs $(z<1.2)$ \\
\hline Carballo et al. (1999) & 48 & $-0.40 \pm 0.14$ & $1300-4500 \AA$ & B3-VLA(radio) QSOs $(z>1.2)$ \\
\hline Vanden Berk et al. (2001) & 2200 & -0.44 & $1300-5000 \AA$ & $\operatorname{SDSS}(z=0.044-4.78)$ \\
\hline Brotherton et al. (2001) & 657 & -0.46 & $1220-4800 \AA$ & FBQS (First Bright Quasar Survey) \\
\hline Telfer et al. (2002) & 77 & $-1.57 \pm 0.17$ & $500-1200 \AA$ & $H S T / F O S$ (radio-quiet sample) \\
\hline Telfer et al. (2002) & 107 & $-1.96 \pm 0.12$ & $500-1200 \AA$ & $H S T / F O S$ (radio-loud sample) \\
\hline Scott et al. (2004) & 85 & $-0.56_{-0.28}^{+0.38}$ & $630-1155 \AA$ & FUSE ultraviolet $(z<0.67)$ \\
\hline This paper & 15 & $-1.41 \pm 0.21$ & $550-1000 \AA$ & $H S T /$ COS ultraviolet $(0.45<z<1.44)$ \\
\hline This paper & 7 & $-0.68 \pm 0.14$ & $1200-1750 \AA$ & $H S T / \mathrm{COS}$ ultraviolet $(z<0.16)$ \\
\hline
\end{tabular}

a Top group of surveys sample visible/FUV wavelengths $(\lambda>1200 \AA)$, while lower group samples EUV $(\lambda<1000 \AA)$. The columns list: composite survey reference; mean or median spectral index; number of QSOs in survey; rest-wavelength range of fit; survey characteristics.

TABLE 4

AGN EMission Lines AND Blends (FUV AND EUV)

\begin{tabular}{|c|c|c|}
\hline Ion & Wavelength $^{\mathrm{a}}$ & Comments $^{\mathrm{a}}$ \\
\hline $\mathrm{N}$ III] & 1750.2 & Semi-forbidden lines $\left[2 \mathrm{~s} 2 \mathrm{p}^{2}\left({ }^{4} \mathrm{P}\right) \rightarrow 2 \mathrm{~s}^{2} 2 \mathrm{p}\left({ }^{2} \mathrm{P}\right)\right]$ (blend of $\left.1749.7,1752.2\right)$ \\
\hline O III & 1664.7 & Semi-forbidden lines $\left[2 \mathrm{~s} 2 \mathrm{p}^{3}\left({ }^{5} \mathrm{~S}\right) \rightarrow 2 \mathrm{~s}^{2} 2 \mathrm{p}^{2}\left({ }^{3} \mathrm{P}\right)\right]$ (blend of $1666.2,1660.8$ ) \\
\hline He II & 1640.5 & He II (Balmer- $\alpha$ ) line \\
\hline C IV & 1549.0 & Permitted doublet $\left[2 \mathrm{p}\left({ }^{2} \mathrm{P}\right) \rightarrow 2 \mathrm{~s}\left({ }^{2} \mathrm{~S}\right)\right]$ (blend of $1548.19,1550.77$ ) \\
\hline $\mathrm{N}$ IV] & 1486.5 & Semi-forbidden line $\left[2 \mathrm{~s} 2 \mathrm{p}\left({ }^{3} \mathrm{P}_{1}\right) \rightarrow 2 \mathrm{~s}^{2}\left({ }^{1} \mathrm{~S}_{0}\right)\right]$ \\
\hline O IV] & 1404.8 & Semi-forbidden lines (blend of $1407.4,1404.8,1401.2$ ) \\
\hline Si IV & 1396.75 & Permitted doublet $\left[3 \mathrm{p}\left({ }^{2} \mathrm{P}\right) \rightarrow 3 \mathrm{~s}\left({ }^{2} \mathrm{~S}\right)\right]$ (blend of $1393.76,1402.77$ ) \\
\hline C II & 1335.31 & Three permitted lines (blend of $1334.43,1335.66,1335.71$ ) \\
\hline Si II & 1307.64 & Permitted doublet (blend of $1304.37,1309.28$ ) \\
\hline $\mathrm{O}$ I & 1303.49 & Three permitted lines (blend of $1302.17,1304.86,1306.03$ ) \\
\hline Si II & 1263.31 & Three permitted lines (blend of $1260.42,1264.74,1265.00$ ) \\
\hline $\mathrm{N}$ V & 1240.15 & Permitted doublet $\left[2 \mathrm{p}\left({ }^{2} \mathrm{P}\right) \rightarrow 2 \mathrm{~s}\left({ }^{2} \mathrm{~S}\right)\right]$ (blend of $1238.82,1242.80$ ) \\
\hline $\mathrm{H} \mathrm{I}$ & 1215.67 & Permitted H I (Lyman- $\alpha)$ line \\
\hline C III & 1175.25 & Subordinate line $\left[2 \mathrm{p}^{2}\left({ }^{3} \mathrm{P}\right) \rightarrow 2 \mathrm{~s} 2 \mathrm{p}\left({ }^{3} \mathrm{P}\right)\right]$ \\
\hline Fe III & 1125.79 & Many lines $(1122.53-1131.91)$ from $\left[3 \mathrm{~d}^{5} 4 \mathrm{p}\left({ }^{5} \mathrm{P}\right) \rightarrow 3 \mathrm{~d}^{6}\left({ }^{5} \mathrm{D}\right)\right]$ \\
\hline $\mathrm{N}$ II & 1085.12 & Many lines $(1083.99-1085.70)$ from $\left[2 \mathrm{~s} 2 \mathrm{p}^{3}\left({ }^{3} \mathrm{D}\right) \rightarrow 2 \mathrm{~s}^{2} 2 \mathrm{p}^{2}\left({ }^{3} \mathrm{P}\right)\right]$ \\
\hline $\mathrm{O} \mathrm{VI}$ & 1033.82 & Permitted doublet $\left[2 \mathrm{p}\left({ }^{2} \mathrm{P}\right) \rightarrow 2 \mathrm{~s}\left({ }^{2} \mathrm{~S}\right)\right]$ (blend of $1031.93,1037.62, \mathrm{H}$ I Ly $\beta 1025.72$ ) \\
\hline N III & 990.98 & Three permitted lines (blend of $989.80,991.51,991.58$ ) \\
\hline C II & 903.8 & Two permitted lines $\left[2 \mathrm{~s} 2 \mathrm{p}^{2}\left({ }^{2} \mathrm{P}\right) \rightarrow 2 \mathrm{~s}^{2} 2 \mathrm{p}\left({ }^{2} \mathrm{P}\right)\right]$ \\
\hline O II & 833.8 & Three permitted lines $\left[2 \mathrm{~s} 2 \mathrm{p}^{4}\left({ }^{4} \mathrm{P}\right) \rightarrow 2 \mathrm{~s}^{2} 2 \mathrm{p}^{3}\left({ }^{4} \mathrm{~S}\right)\right]$ \\
\hline O III & 832.9 & Three permitted lines $\left[2 \mathrm{~s} 2 \mathrm{p}^{3}\left({ }^{3} \mathrm{D}\right) \rightarrow 2 \mathrm{~s}^{2} 2 \mathrm{p}^{2}\left({ }^{3} \mathrm{P}\right)\right]$ \\
\hline O IV & 787.7 & Three permitted lines $\left[2 \mathrm{~s} 2 \mathrm{p}^{2}\left({ }^{2} \mathrm{D}\right) \rightarrow 2 \mathrm{~s}^{2} 2 \mathrm{p}\left({ }^{2} \mathrm{P}\right)\right]$ \\
\hline Ne VIII & 773.7 & Doublet $\left[2 \mathrm{p}\left({ }^{2} \mathrm{P}\right) \rightarrow 2 \mathrm{~s}\left({ }^{2} \mathrm{~S}\right)\right]$ (blend of $770.41,780.32$ ) \\
\hline N IV & 765.1 & Permitted line $\left[2 \mathrm{~s} 2 \mathrm{p}\left({ }^{1} \mathrm{P}_{1}\right) \rightarrow 2 \mathrm{~s}^{2}\left({ }^{1} \mathrm{~S}_{0}\right)\right]$ \\
\hline O III & 702.3 & Permitted lines $\left[2 \mathrm{~s}^{3} \mathrm{p}^{3}\left({ }^{3} \mathrm{P}\right) \rightarrow 2 \mathrm{~s}^{2} 2 \mathrm{p}^{2}\left({ }^{3} \mathrm{P}\right)\right]$ \\
\hline $\mathrm{N}$ III & 685.5 & Permitted lines $\left[2 \mathrm{~s} 2 \mathrm{p}^{2}\left({ }^{2} \mathrm{P}\right) \rightarrow 2 \mathrm{~s}^{2} 2 \mathrm{p}\left({ }^{2} \mathrm{P}\right)\right]$ \\
\hline O IV & 608.4 & Permitted lines $\left[2 \mathrm{~s} 2 \mathrm{p}^{2}\left({ }^{2} \mathrm{~S}\right) \rightarrow 2 \mathrm{~s}^{2} 2 \mathrm{p}\left({ }^{2} \mathrm{P}\right)\right]$ \\
\hline O IV & 554.1 & Permitted lines $\left[2 \mathrm{~s} 2 \mathrm{p}^{2}\left({ }^{2} \mathrm{P}\right) \rightarrow 2 \mathrm{~s}^{2} 2 \mathrm{p}\left({ }^{2} \mathrm{P}\right)\right]$ \\
\hline $\mathrm{Ne} V$ & 569.7 & Permitted lines $\left[2 \mathrm{~s} 2 \mathrm{p}^{3}\left({ }^{3} \mathrm{D}\right) \rightarrow 2 \mathrm{~s}^{2} 2 \mathrm{p}^{2}\left({ }^{3} \mathrm{P}\right)\right]$ \\
\hline $\mathrm{O} \mathrm{V}$ & 629.7 & Permitted line $\left[2 \mathrm{~s} 2 \mathrm{p}\left({ }^{1} \mathrm{P}_{1}\right) \rightarrow 2 \mathrm{~s}^{2}\left({ }^{1} \mathrm{~S}_{0}\right)\right]$ \\
\hline
\end{tabular}

${ }^{a}$ Permitted and semi-forbidden lines, configurations, transitions, blends, and statistically weighted wavelengths. Data sources: Morton (2003), Verner et al. (1994), and NIST Data Tables. 